\section{Cahiers de Narratologie}

Analyse et théorie narratives

33 | 2018

L'Art du roman chez Umberto Eco

\title{
L'apathique et l'esthétique. Réflexions sur les clausules du Nom de la rose et du Pendule de Foucault d'Umberto Eco
}

Piero Polidoro

\section{OpenEdition}

Journals

Electronic version

URL: http://journals.openedition.org/narratologie/8304

DOI: $10.4000 /$ narratologie.8304

ISSN: 1765-307X

Publisher

LIRCES

\section{Electronic reference}

Piero Polidoro, «L'apathique et l'esthétique. Réflexions sur les clausules du Nom de la rose et du Pendule de Foucault d'Umberto Eco », Cahiers de Narratologie [Online], 33 | 2018, Online since 03 July 2018, connection on 01 May 2019. URL : http://journals.openedition.org/narratologie/8304; DOI : 10.4000/narratologie.8304

This text was automatically generated on 1 May 2019.

Article L.111-1 du Code de la propriété intellectuelle. 


\title{
L'apathique et l'esthétique. Réflexions sur les clausules du Nom de la rose et du Pendule de Foucault d'Umberto Eco
}

\author{
Piero Polidoro
}

1 Dans cet article, je voudrais proposer une lecture de la clausule du deuxième roman d'Umberto Eco, Le pendule de Foucault (1988). A cette fin, je la comparerai à celle de son premier roman, Le Nom de la rose (1980), en soulignant analogies et différences et en tenant compte de sa pensée théorique. Je veux suivre la leçon d'Eco et donc je ne vais pas chercher de reconstruire l'intentio auctoris (Eco 1990), c'est-à-dire le sens que l'auteur voulait donner au texte, une question peu ou pas du tout intéressante en sémiotique. Mon but est plutôt d'identifier une intentio operis, donc une possible ligne interprétative qui soit cohérente avec le texte et avec le corpus constitué par les œuvres (théoriques et narratives) d'Eco ; et j'espère bien que ce ne sera pas une dérive interprétative (intentio lectoris) d'un lecteur illuminé qui voulait trouver dans le texte quelque chose qu'il n'y avait pas.

\section{Les domaines de la théorie et de la narration chez Umberto Eco}

Dans l'œuvre d'Umberto Eco, l'activité de théoricien de la sémiotique et celle de narrateur sont liées étroitement.

3 C'est Eco lui-même qui a écrit plusieurs fois, en qualité de théoricien, sur son activité de narrateur; on peut rappeler la célèbre Apostille au « Nom de la rose » (1983) ou les articles publiés dans les livres De la littérature (2002) et Confessions d'un jeune romancier (2011). Mais si ce genre d'écrits montre clairement comment Eco le sémioticien a traité d'Eco le romancier, il faut encore démontrer l'existence du parcours inverse, c'est-à-dire 
comment le romancier a écrit sur des thèmes qui étaient typiques du sémioticien. Une certaine continuité, ou mieux osmose, entre les deux domaines est évidente: dans les années pendant lesquelles il était en train d'écrire Le Nom de la Rose (1978-1979, selon Eco 2002 : 347), Eco publia ce qui restera son seul livre complétement dédié à la sémiotique narrative: Lector in fabula (1979); son deuxième roman, Le Pendule de Foucault, est contemporain des séminaires sur l'hermétisme organisés par Eco à l'Université de Bologne et à plusieurs essais qui en 1990 ont été collectés dans le livre Les Limites de l'interprétation; et les exemples pourraient continuer.

4 Récemment Claudio Paolucci, un disciple d'Eco, a traité des contacts entre théorie et narration dans l'œuvre de son maitre dans un livre à lui dédié et publié en italien en 2017. Selon Paolucci il y a un fil rouge qui franchit la pensée d'Eco : c'est le chevauchement et la connexion entre domaines différents. "Per risolvere un problema che si è ben posto - determinando il suo dominio di appartenenza e la sua posizione interna a quel dominio - occorre per essenza uscire fuori da quel dominio, operando traduzioni e concatenamenti con domini eterogenei» (Paolucci 2017: 56; "Pour résoudre une question qui a été bien posée - en déterminant son domaine d'appartenance et sa position dans ce domaine - il faut par essence sortir dehors ce domaine, en opérant traductions et enchaînements avec des domaines hétérogènes ", ma traduction). C'est exactement ce qui se passe, par exemple, dans le premier essai d'Eco, L'œuvre ouverte, dans lequel on comprend le domaine de l'art contemporain et des avant-gardes du deuxième aprèsguerre en sortant du domaine de l'art et en s'aventurant dans celui de sciences comme la physique et la mathématique. C'est à partir de cette idée du chevauchement et de l'éclaircissement réciproque entre domaines différents que Paolucci lit le rapport entre théorie et narration chez Eco.

Dans son autobiographie intellectuelle, écrite pour le volume de la Library of Living Philosophers qu'on lui a consacré (Beardsworth et Auxier, 2017), Eco ne s'attarde pas beaucoup sur son activité de romancier, mais il écrit une chose très intéressante, en paraphrasant Wittgenstein :

The jacket flap of the first Italian edition [of the Name of the Rose] said, in conclusion : «If the author has written a novel is because he has discovered, in his maturity, that what you cannot theorize about, you must narrate ». (Eco 2017: 58; «La quatrième de couverture de la première édition [du Nom de la rose] disait, dans sa conclusion: "Si l'auteur a écrit un roman, c'est pourquoi il a découvert, à l'âge de la maturité, que ce dont on ne peut pas parler, il faut le narrer" ", c'est nous qui traduisons)

Paolucci commence par cette affirmation pour expliquer la relation entre l'activité théorique et l'activité narrative chez Eco. Dans l'interprétation de la pensée d'Eco donnée par Paolucci, la théorie est toujours supérieure à la narration, parce que cette dernière n'est qu'un type de langage et, donc, elle peut être étudiée par la théorie. D'ailleurs, la narration est une forme d'expression particulière. Même si la théorie dépasse la narration, celle-ci est une forme d'expression qui a des potentialités différentes (attention: différentes, et non supérieures ou inférieures) de celles de la théorie et donc elle peut dire des choses qui sont indicibles dans la théorie.

Eco ha insistito in più punti sul fatto che la sua attività di romanziere sia nata dell'esigenza di esprimere qualcosa che non si poteva rappresentare attraverso la struttura del teorizzare. C'era evidentemente la sensazione che qualcosa non potesse essere espresso, ma non potesse essere espresso all'interno di quel sistema di espressione. È come se la struttura di quel particolare dominio non fosse adatta all'espressione di quella particolare esperienza e ne servissero altri. Tuttavia, al di 
fuori di quel particolare dominio non si trovavano l'energia creatrice e il vortice dionisiaco con le sue forze su cui l'analisi non ha presa; bensì altre strutture, di forma differente e per questo più adatte all'espressione di quel tipo particolare di esperienza. (Paolucci 2017: 133; «Eco a insisté plusieurs fois sur le fait que son activité de romancier est née de l'exigence d'exprimer quelque chose qui ne pouvait pas être représentée par les structures de la théorisation. Il avait, évidemment, la sensation que quelque chose ne pouvait pas être exprimé, mais ne pouvait pas être exprimé dans ce système de l'expression. C'est comme si la structure de ce domaine particulier n'était pas adaptée à l'expression de cette expérience particulière et qu'il en fallait d'autres. Toutefois, en dehors de ce domaine particulier il n'y avait pas l'énergie créatrice et le tourbillon dionysiaque avec ses forces sur lesquelles l'analyse n'a pas de saisie; mais il y a d'autres structures, avec une forme différente et pour ça plus adaptées à l'expression de ce type particulier d'expérience", c'est nous qui traduisons)

7 Dans un autre passage très intéressant de son livre, Paolucci analyse ce rapport d'un autre point de vue. Comme on le sait, à partir du Trattato di semiotica generale, Eco a souvent dit que les signes peuvent être utilisés pour mentir et donc la sémiotique ne s'occupe pas de la vérité.

Al contrario, è la Verità che arriva a un certo punto a supplire ad alcune impossibilità proprie dei segni, a mostrare cose che i segni non riescono per essenza a dire. In Eco la verità non può essere detta, può soltanto essere mostrata, a un certo livello. [...] Teoria, interpretazione e congettura servono a costruire versioni del mondo reale e possono sempre venire utilizzate per mentire. Al contrario, il mondo della Verità rimanda invece alla narrazione, alla Necessità, all'ineluttabilità di qualcosa che è, che è stato e che non può essere altrimenti. (Paolucci $2017: 25 ;$ « Au contraire, c'est la Vérité qui arrive - à un certain moment - pour suppléer aux impossibilités typiques des signes, pour montrer des choses que les signes ne peuvent pas - par essence - dire. Chez Eco, la vérité ne peut pas être dite, elle peut seulement être montrée, à un certain niveau. [...] Théorie, interprétation et conjecture servent à construire des versions du monde réel et peuvent toujours être utilisées pour mentir. Au contraire, le monde de la Vérité renvoie à la narration, à la Nécessité, à l'inéluctabilité de quelque chose qui est, qui a été et qui ne peut pas être autrement. ", c'est nous qui traduisons)

Les réflexions de Paolucci nous offrent un cadre intéressant pour interpréter les rapports entre théorie et narration dans la pensée d'Eco et, comme on verra, elles seront très utiles dans ce qui suit.

\section{La clausule du Nom de la rose}

9 Dans les deux chapitres dédiés au septième et dernier jour de l'histoire du Nom de la rose, se développe la clausule du roman. Guillaume de Baskerville et son adjuvant Adso de Melk ont réussi à s'avancer dans la zone plus secrète de la bibliothèque et là, ils ont trouvé - comme Guillaume avait déjà deviné - Jorge de Burgos, le vieux moine aveugle qui est le responsable - direct ou indirect - de beaucoup des morts qui sont arrivées dans l'abbaye. Le but de Jorge est de cacher l'existence d'un manuscrit, la dernière copie au monde du deuxième livre de la Poétique d'Aristote, qu'il considère comme dangereux parce qu'il exalte le rire et donc il priverait les êtres humains de la crainte de Dieu. A la fin d'un intense échange dialectique, Jorge et Guillaume se heurtent, se battent pour le manuscrit, la lanterne tombe et met feu aux parchemins. L'incendie se répand vite et, en l'espace de peu de temps, il détruit la bibliothèque et l'abbaye. Dans les dernières pages de ces chapitres, nous trouvons Guillaume et Adso qui assistent au désastre, impuissants, parce 
qu'ils sont désormais épuisés par les vaines tentatives d'éteindre l'incendie et de sauver les manuscrits.

Guillaume, dévasté, reconnait que les hypothèses qu'il avait formulées pour résoudre les délits se sont avérées fausses, même si grâce à elles il a dévoilé le mystère.

Dove sta tutta la mia saggezza? Mi sono comportato da ostinato, inseguendo una parvenza di ordine, quando dovevo sapere bene che non vi è un ordine nell'universo. [...] L'ordine che la nostra mente immagina è come una rete, o una scala, che si costruisce per raggiungere qualcosa. Ma dopo si deve gettare la scala, perché si scopre che, se pure serviva, era priva di senso. [...] È difficile accettare l'idea che non vi può essere un ordine nell'universo, perché offenderebbe la libera volontà di Dio e la sua onnipotenza. Così la libertà di Dio è la nostra condanna, o almeno la condanna della nostra superbia. (Eco 1980: 495; «Où gît toute ma sagesse ? Je me suis comporté en homme obstiné, poursuivant un simulacre d'ordre, quand je devais bien savoir qu'il n'est point d'ordre dans l'univers. [...] L'ordre que notre esprit imagine est comme un filet, ou une échelle, que l'on construit pour atteindre quelque chose. Mais après, on doit jeter l'échelle, car l'on découvre que, si même elle servait, elle était dénuée de sens. [...] Il est difficile d'accepter l'idée qu'il ne peut y avoir un ordre dans l'univers, parce qu'il offenserait la libre volonté de Dieu et son omnipotence. Ainsi la liberté de Dieu est notre condamnation, ou du moins la condamnation de notre superbe », Eco 1982 : 614-615)

Face aux mots de Guillaume, Adso demande :

« Ma come può esistere un essere necessario totalmente intessuto di possibile ? Che differenza c'è allora tra Dio e il caos primigenio ? Affermare l'assoluta onnipotenza di Dio e la sua assoluta disponibilità rispetto alle sue stesse scelte, non equivale a dimostrare che Dio non esiste?».

Guglielmo mi guardò senza che alcun sentimento trasparisse dai tratti del suo viso, e disse: "Come potrebbe un sapiente continuare a comunicare il suo sapere se rispondesse di sì alla tua domanda? ». (Eco 1980 : 496)

( "Mais comment peut exister un être nécessaire totalement tissu de possible? Quelle différence y a-t-il alors entre Dieu et le chaos originel? Affirmer l'omnipotence absolue de Dieu et son absolue disponibilité en regard de ses choix mêmes, n'équivaut-il pas à démontrer que Dieu n'existe pas ?"

Guillaume me regarda sans qu'aucun sentiment filtrât des linéaments de son visage, et il dit: "Comment un savant pourrait-il continuer à communiquer son savoir s'il répondait oui à ta question?" ", Eco $1982: 615$

Ce sont presque les derniers mots dits par Guillaume et cités dans le roman. Dans le bref chapitre final, « Dernier Feuillet », est narrée la séparation finale entre Adso et son maître Guillaume. Adso ajoute que Guillaume mourut pendant la Peste noire qui frappa l'Europe dans la moitié du XIVème siècle. Nous, lecteurs, pouvons imaginer les dernières années de sa vie, un homme vaincu dans sa vision intellectuelle et politique. Le roman, comme on le sait, se conclut avec un hexamètre pris du De comptemptu mundi de Bernard de Cluny: «stat rosa pristina nomine, nomina nuda tenemus ».

\section{Sémiotique, réalisme, nominalisme, nihilisme}

Les dernières pages du Nom de la rose et surtout l'hexamètre final ont bien contribué à donner au roman la réputation d'être un hymne au nominalisme et même au nihilisme. La critique la plus extrême faite à Eco vient du Père Guido Sommavilla dans un commentaire (devenu célèbre, au moins dans le paysage culturel italien) publié au mois de septembre 1981 dans la revue jésuite La civiltà cattolica. Le titre du commentaire est très explicatif: «L'allegro nominalismo nichilistico di Umberto Eco» (« Le joyeux nominalisme nihiliste 
d'Umberto Eco »). Les accusations que l'auteur adressait à Eco et à son roman étaient donc doubles et complémentaires : tout d'abord en le traitant de nominaliste, c'est-à-dire - du point de vue de Sommavilla - penser que l'être humain, en ayant seulement le langage, ne peut pas rejoindre aucune vérité sinon celle de l'absence de la vérité ; seconde accusation (et conséquente), de soutenir une position nihiliste et donc l'annulation de toutes les différences et du sens fondé.

Il secondo libro della Poetica di Aristotele] avrebbe insegnato a riconoscere la sostanza ridicola di tutta la realtà, a confondere i valori più alti con i più bassi, $\mathrm{e}$ dunque a ridere dei primi come dei secondi [...] Era insomma un Aristotele, tutto romanzesco evidentemente, che aveva già intravisto la "verità » nominalisticonichilistica di Umberto Eco, la verità che non esiste nulla di serio.

Precisamente questa era anche la « verità » che Guglielmo cercava, che già in fondo sapeva. [...]

La vera verità di Guglielmo-Eco è non meno evidentemente quella di una inquisizione «illuministica » e poi inoltre «nominalistica », protesa ad abbattere la prima [la verità di Jorge] con l'abbattimento di ogni possibile verità, con il dogma dei nuda nomina. (Sommavilla $1981: 505$ )

(" [Le deuxième livre de la Poétique d'Aristote] aurait enseigné à reconnaître la substance ridicule de toute réalité, à confondre les valeurs les plus hautes avec celles les plus basses, et donc à rire des premières comme des secondes [...] C'était, enfin, un Aristote - tout romanesque, évidemment - qui avait déjà entrevu la vérité nominaliste-nihiliste d'Umberto Eco, la vérité que rien de sérieux n'existe.

Précisément, c'était la même "vérité" que Guillaume cherchait, que - dans l'ensemble - il connaissait déjà. [...]

La vraie "vérité" de Guillaume-Eco est, pas moins évidemment, celle d'une inquisition "illuministe" et en outre "nominaliste", tendant à abattre la première [la vérité de Jorge] avec la destruction de toutes les vérités possibles, avec le dogme des nuda nomina. », c'est nous qui traduisons)

Sommavilla ne fut pas le seul à adresser une telle accusation (si on peut parler d'accusation) à Eco et à son roman. Utilisant un ton beaucoup plus modéré, d'autres critiques parlaient de nominalisme, par exemple Huguette Hatem (1982), et Vintila Horia (1983) parla du Nom de la rose comme d'un roman polythéiste.

Mais l'interprétation nihiliste de la pensée d'Eco n'est pas soutenue par ses œuvres ; et, en effet, dans les mêmes années, il était en train de développer sa forme particulière de réalisme, dont je parlerai bientôt.

Pour ce qui concerne Le nom de la rose et sa phrase finale, on peut reprendre ce qui a été écrit par Paolucci dans le livre que j'ai déjà cité. Tout d'abord, Paolucci rappelle que c'est juste Eco qui nous a enseigné à ne pas confondre l'intentio operis (ici une citation nominaliste faite par Adso à la fin de son conte) et l'intentio auctoris (la position philosophique d'Eco sur ces thèmes). Il souligne aussi que c'est très difficile d'associer au nominalisme et surtout au nihilisme un auteur qui a édifié sa réflexion sémiotique sur la pensée de Charles Sanders Peirce et qui, au moins à partir des années soixante-dix, a toujours pratiqué un « réalisme scotiste ». Ce que, selon Paolucci, Eco voulait dire à la fin de son roman est que

[...] i) noi possiamo accedere alle cose solamente attraverso la mediazione dei segni ;

ii) i segni possono sempre venire utilizzati per mentire. Per questo l'acceso alla verità è sempre potenzialmente fallibile e condizionato a una semiotica, che sia in grado di fare perizia sulla trama dei segni in oggetto. (Paolucci $2017: 149$ )

(« [...] i) nous ne pouvons pas accéder aux choses seulement à travers la médiation des signes; ii) les signes peuvent toujours être utilisés pour mentir. C'est pour ça que l'accès à la vérité est toujours potentiellement faillible et conditionné à une 
sémiotique, qui soit capable de faire expertise sur la trame des signes en objet. ", c'est nous qui traduisons)

\section{Le réalisme négatif}

17 A la fin du paragraphe précédent, j'ai mentionné la forme particulière de réalisme épousée par Eco ; cette conception commençait à émerger dans ses écrits dans les mêmes années pendant lesquelles Le nom de la rose, une fois publié, connaissait un succès croissant ; Eco réaffirma cela de façon plus nuancée dans Kant et l'ornithorynque (Eco 1997) et le reformula de manière encore plus claire, pendant les dernières années de sa vie, alors que la communauté philosophique commençait à être parcourue par un débat renouvelé sur le réalisme. Le but de cet article n'est pas d'illustrer ou de discuter le réalisme chez Eco. C'est pour cela d'ailleurs que je me limiterai ici à synthétiser les principaux aspects de cette théorie et de celles qui sont plus liées à la thèse que je vais soutenir.

18 Eco a plusieurs fois parlé de son réalisme comme d'un « réalisme négatif » (Eco 2012). Le point nodal de cette position est très bien clarifié dans Kant et l'ornithorynque (Eco 1997). Ici, Eco n'abandonne pas l'idée que la culture et les systèmes sémiotiques qui la constituent jouent un rôle fondamental dans la construction de la réalité. Mais ce qu'il nie est que ce pouvoir constructif soit absolu. Contre les interprétations extrêmes du structuralisme hjelmslevien, selon lesquelles la grille constituée par le langage bénéficierait d'une pleine autonomie pour découper (et donc pour représenter) sous des formes différentes la réalité, Eco parle de l'existence d'un « socle dur » de l'être. Ce socle dur ne détermine pas la construction de la réalité (comme pourraient penser un réaliste ingénu ou un réaliste "positif »), mais quand même il offre une résistance. Selon une autre métaphore utilisée par Eco, ce socle dur est traversé par des nervures, comme celles qui traversent le marbre et qui imposent de le découper de certaines façons, mais pas de toutes les façons. De même, les nervures de l'être acceptent certaines formes sémiotiques, mais elles en refusent d'autres. On pourrait dire que l'être n'oblige pas, mais il interdit : il ne nous dit pas quelle forme utiliser pour le décrire, mais il indique les formes qui ne sont pas admises. D'ici l'expression « réalisme négatif ».

19 Il semble évident que ceci n'est pas une position nihiliste. Les positions nihilistes sont celles qui, s'inspirant de Nietzsche, partent de la présupposition qu'il n'y a pas de faits, mais seulement des interprétations. Toutefois, Eco (2017: 50) observe que dire qu'il y a seulement des interprétations n'exclut pas qu'il puisse y avoir de mauvaises interprétations. Son idée est tout à fait différente, comme il l'a expliqué récemment dans son autobiographie intellectuelle.

If the continuum itself has lines of tendency we are not entitled to say whatever we like. There are directions, maybe not compulsory directions but certainly directions that are forbidden. There are things we cannot say. (Eco $2017: 54$ )

( $\mathrm{Si}$ le continuum a des lignes de tendance, nous n'avons pas le droit de dire n'importe quoi. Il y a des directions, peut-être pas des directions obligatoires, mais certainement des directions qui sont interdites. Il y a des choses que nous ne pouvons pas dire.) (c'est nous qui traduisons)

D'ailleurs, presque dix ans avant Kant et l'ornithorynque et bien des années avant le retour du réalisme au centre du débat philosophique, Eco avait déjà exprimé cette idée très clairement dans son deuxième roman, Le Pendule de Foucault :

E questo vuole dire che egli in qualche modo sapeva che, per fragile che l'essere sia, per infinita e senza scopo che sia la nostra interrogazione del mondo, c'è qualcosa 
che ha più senso del resto. (Eco $1988: 659$ )

(«En somme, d'une certaine façon, il savait que, pour fragile que soit l'être, pour infinie et sans but que soit notre interrogation du monde, il existe quelque chose qui a plus de sens que le reste. », Eco $1990: 632$ )

Dans ce magma indistinct qui peut nous sembler chaos, absence d'ordre (comme il arrive à Guillaume face à la destruction de la bibliothèque), émergent quand-même des lignes, quelque chose qui a plus sens que le reste. C'est évident que tout ceci contraste avec l'intention attribuée à Eco par Sommavilla, selon laquelle l'auteur visait à confondre les distinctions entre valeurs, à ne considérer rien sérieusement. Au contraire, déjà dans les années soixante-dix, Eco allait dans la direction opposée, l'a été très bien souligné par Mohamed Bernoussi en parlant de Kant et l'ornithorynque et de ce qui l'avait précédé.

Umberto Eco renoue ici avec une conviction chère développée depuis le début et annoncée de façon forte dans le Trattato, à savoir lier une réflexion philosophique à une véritable philosophie de l'action ou de l'engagement. "Le realist turn ", nous semble-t-il, est l'aboutissement d'une longue et tenace lutte pour ancrer la sémiosis dans la société. (Bernoussi 2017 : 105)

\section{Une théorie raisonnable}

Comme je l'ai expliqué plus largement dans un essai publié en 2017 (Polidoro 2017), je crois que le réalisme négatif est un des aspects d'une caractéristique transversale de la pensée d'Eco : la raisonnabilité. Le terme "raison » indique soit une faculté générale de l'être humain qui conduit notre activité d'investigation et de raisonnement, soit une manière spécifique d'action de cette faculté (par exemple, la raison comme procédure discursive). Le terme "raisonnabilité", par contre, a une connotation de limite; il indique la présence d'un doute sur l'infaillibilité de la raison; ou la restriction de la validité d'une argumentation à un certain domaine ou à une certaine circonstance.

Mais la raisonnabilité ne doit pas être confondue avec une "pensée faible », nihiliste. Elle est dotée des instruments adaptés pour ne pas tomber dans une impasse. Par exemple, la position raisonnable d'Eco dans le "débat sur l'iconisme " (Polidoro 2012, 2015), qui voyait opposés les défenseurs d'une conception « naturaliste » des signe iconiques et - de l'autre côté - les conventionnalistes les plus extrêmes, était celle de trouver une synthèse et d'utiliser des concepts qui n'étaient pas nets, mais complexes et hybrides. Ainsi, dans Kant et l'ornithorynque, Eco revient sur ce débat et il clarifie la position intermédiaire qu'il avait soutenue depuis longtemps; pour la souligner, il indique les signes iconiques avec un terme impur, diminué et donc - de mon point de vue - raisonnable : «hypoicone». Comme hypoicones, les signes iconiques ne sont ni totalement naturels ni totalement arbitraires, mais un mélange de motivation et de conventionalité. Toujours sous le signe de la raisonnabilité, quelques années avant, dans Les limites de l'interprétation, Eco avait montré comment arrêter la dérive interprétative qu'on aurait pu justifier (à tort) à la lumière de ce qu'il avait écrit dans L'œuvre ouverte. Et la solution venait, dans cette situation aussi, d'une approche négative: on ne peut pas dire quelle est la meilleure interprétation d'un texte, mais on peut certainement dire quelles sont les mauvaises.

Ainsi, même dans l'opposition entre le réalisme aristotélicien et le dogme de l'arbitraire et d'une forme sémiotique totalement autonome vis-à-vis de la réalité (qui peut conduire à un extrême idéalisme), le réalisme négatif proposé par Eco offre une solution raisonnable. Mais elle peut se réaliser seulement si on est prêt à accepter un "point d'ancrage ", un point d'appui, peut-être pas facilement accessible, mais sur lequel 
construire tout le reste. Le socle dur de l'être, les nervures du marbre représentent ce point d'ancrage, que la réalité nous offre pour construire une théorie de la connaissance qui ne soit pas obligée de se rendre au désordre du monde ou à son éloignement des formes sémiotiques que nous construisons pour le décrire. Un point d'ancrage, enfin, pour ne pas sombrer dans la méfiance et l'apathie que Guillaume semble ressentir au moment où il se rend compte de sa défaite gnoséologique.

La raisonnabilité ne nous offre pas une démonstration absolue et complète; mais la raison inflexible et conséquentielle n'est pas capable de le faire non plus. D'un côté, la raisonnabilité nous aide, avec humilité, à nous plier à la nécessité de quelque point d'ancrage qui fonde notre système. C'est partant de ces considérations que je voudrais maintenant proposer une lecture de la clausule du Pendule de Foucault.

\section{La clausule du Pendule de Foucault}

L'épilogue du Pendule de Foucault est constitué par les trois dernières parties du roman. Dans la huitième (et antépénultième), «Hod », Casaubon, caché dans le Conservatoire de Paris, assiste à une cérémonie, pendant laquelle les adeptes de la secte cherchent à arracher un secret (qui n'existe pas) à son ami Belbo, qui refuse de parler et sera tué pour ça. Casaubon arrive à fuir et, après avoir vagué à travers un Paris spectral, rentre en Italie. Dans la pénultième partie, "Jesod», formée de deux chapitres, Casaubon, de nouveau à Milan, décide de se rendre dans le petit village du Piémont où il $\mathrm{y}$ a la maison de campagne de Belbo (chapitre 118). Ici (chapitre 119) il trouve un manuscrit dans lequel Belbo raconte un épisode qu'il a vécu lorsqu'il était jeune, vers la fin de la deuxième guerre mondiale. Belbo doit jouer de la trompette pendant l'enterrement d'un partisan et ce sera son moment de gloire ; pour lui c'est une extase et il cherche à le faire durer le plus longtemps possible. Casaubon comprend qu'il s'agissait de la Grande Occasion de la vie de Belbo, qui malheureusement à l'époque ne pouvait pas se rendre compte que c'était le moment décisif. Casaubon ajoute, dans un passage qui est aussi à l'origine des réflexions de Paolucci que j'ai déjà citées :

Quel giorno Jacopo Belbo aveva fissato negli occhi la Verità. L'unica che gli sarebbe stata concessa, perché la verità che stava apprendendo è che la verità è brevissima (dopo, è solo commento). (Eco $1988: 671$ )

( « Ce jour-là, Jacopo Belbo avait fixé la Vérité dans les yeux. La seule et unique qui lui serait permise, car la vérité qu'il apprenait c'est que la vérité est très brève (après, elle n'est que commentaire). », Eco $1990: 643$ )

On arrive ainsi à la dernière des dix parties qui composent le roman, «Malkut », qui est formée d'un seul chapitre, le numéro 120. Casaubon a terminé de lire le manuscrit de Belbo et il est désormais convaincu que les adeptes de la secte ont découvert son existence et croient qu'il connaît le secret caché par Belbo ; il est par ailleurs résigné au fait qu'ils ont déjà compris où il est, qu'ils sont en train de l'atteindre et que, une fois qu'ils l'auront trouvé, ils le tueront. Dans l'attente, il s'abandonne à ses réflexions.

Dovrei essere in pace. Ho capito. Non dicevano alcuni di coloro cha la salvezza giunge quando si è realizzata la pienezza della conoscenza?

Ho capito. Dovrei essere in pace. Chi diceva che la pace sorge dalla contemplazione dell'ordine, dell'ordine compreso, goduto, realizzato senza residui, gioia, trionfo, cessazione dello sforzo ? Tutto è chiaro, limpido, e l'occhio si posa sul tutto e sulle parti, e vede come le parti cospirassero al tutto, coglie il centro donde scorre la linfa, il soffio, la radice del perché...

Dovrei essere estenuato dalla pace. Dalla finestra dello studio di zio Carlo guardo la 
collina, e quel poco di luna che sta sorgendo. [...]

Ma perché il capire non mi dà pace? Perché amare il Fato, se ti uccide tanto quanto la Provvidenza e il Complotto degli arconti ? Forse non ho ancora capito tutto, mi manca uno spazio, un intervallo. (Eco $1988: 677-678$ )

(Je devrais être en paix. J'ai compris. Certains d'entre eux ne disaient-ils pas que le salut vient quand s'est réalisée la plénitude de la connaissance?

J'ai compris. Je devrais être en paix. Qui disait que la paix naît de la contemplation de l'ordre, de l'ordre compris, savouré, réalisé sans résidus, joie, triomphe, cessation de l'effort? Tout est clair, limpide, et l'œil se pose sur le tout et sur les parties, et il voit comment les parties concouraient au tout, il saisit le centre d'où coule la sève, le souffle, la racine des pourquoi...

Je devrais être exténué par la paix. Par la fenêtre du bureau de l'oncle Carlo, je regarde la colline, et ce peu de lune qui se lève. [...]

Mais pourquoi comprendre ne me donne pas la paix ? Pourquoi aimer le Fatum, s'il te tue autant que la Providence et que le Complot des Archontes? Sans doute n'ai-je pas encore tout compris, il me manque un espace, un intervalle.) (Eco 1990: 649-650)

A la fin du roman, donc, on retrouve, instamment, un mot que Guillaume avait déjà utilisé dans sa dernière conversation avec Adso : ordre. Guillaume l'avait utilisé quatre fois dans peu de phrases (et Adso une cinquième fois, dans un passage que j'ai omis dans la citation). Casaubon répète le mot à courte distance ( « ... de la contemplation de l'ordre, de l'ordre compris...»), en lui conférant un fort relief. Le mot « ordre » est accompagné par le thème de la connaissance: Guillaume dit « Où gît toute ma sagesse? » et, peu après, «Comment un savant pourrait continuer à communiquer son savoir... »; Casaubon parle de « connaissance » et de compréhension.

28 Entre les deux clausules il y a des analogies fortes, mais aussi des différences intéressantes. Guillaume, à la fin de son histoire, suspecte qu'il n'y a pas un ordre du monde ou que les systèmes que nous construisons pour le reconnaître ne peuvent pas le saisir. Casaubon aussi, lorsqu'il devine que la fin s'approche, commence à raisonner sur l'ordre et la connaissance. Casaubon aussi a compris presque la même chose : qu'un ordre (dans Le Nom de la rose le projet apocalyptique des homicides ou l'ordre même de l'univers, dans Le Pendule de Foucault le Plan des conspirateurs) n'existe pas, alors que tout le monde est en train de le chercher. Mais, au contraire de Guillaume, Casaubon semble penser comprendre cela devrait amener paix, sérénité, acceptation du destin. Et pourtant, il ne peut pas y arriver. Il y a quelque chose qui manque ; peut-être qu'il n'a pas encore tout compris, comme il le dit lui-même.

Qu'est-ce qu'il y a encore à comprendre ? Quelque chose qui se trouve au-delà, ou peutêtre avant la pensée rationnelle, ou le raisonnement articulé. En cela, le parcours de Casaubon diverge de celui de Guillaume. Adso notait que lorsque Guillaume était en train de prononcer la phrase sur l'impossibilité de répondre oui à la question sur l'existence de Dieu, il le regardait «sans qu'aucun sentiment ne filtrât des linéaments de son visage ». Guillaume se rend et dans sa défaite il devient apathique. Par contre, les derniers mots de Casaubon sont riches d'émotions. Au début il attend la paix, la sérénité; mais il ne les trouve pas et il commence à sentir la douleur : « Mi fa male pensare che non vedrò più Lia e il bambino. [...] Merda. Eppure fa male. Pazienza, appena sono morto me lo dimentico » (Eco 1988: 679; « Ça fait mal de penser que je ne verrai plus Lia et le petit [...] Merde. Et pourtant ça fait mal. Patience, à peine je suis mort je l'oublie. ») (Eco 1990 : 650).

Dans le dernier chapitre, donc, la focalisation se déplace graduellement des événements et des raisonnements aux passions. Dans les termes d'un autre théoricien de la 
sémiotique, Algirdas Julien Greimas, on pourrait dire que les niveaux pragmatique (événements) et cognitif (raisonnements) ont laissé la place à celui pathémique (passions). En même temps, on assiste - et pardonnez le calembour - à l'émersion d'un autre sens, celui des sens (entendus comme systèmes sensoriels).

Lungo le falde del Bricco si stendono filari e filari di viti. Li so, ne ho visti di simili ai miei tempi. Nessuna Dottrina dei Numeri ha mai potuto dire se sorgono in salita o in discesa. In mezzo ai filari, ma ci devi camminare scalzo col tallone un po' calloso, sin da piccolo, ci sono degli alberi di pesche. Sono pesche gialle che crescono solo tra i filari, si spaccano con la pressione del pollice, e l'osso ne esce quasi da solo, pulito come dopo un trattamento chimico, salvo qualche vermiciattolo grasso e bianco di polpa che vi rimane attaccato per un atomo. Puoi mangiarle senza quasi sentire il velluto della pelle, che ti fa scorrere i brividi dalla lingua sino all'inguine. Un tempo lì pascolavano i dinosauri. Poi un'altra superficie ha coperto la loro. Eppure, come Belbo nel momento in cui suonava la tromba, quando davo un morso alle pesche capivo il Regno ed ero tutt'uno con lui. Dopo, solo arguzia. Inventa, inventa il Piano, Casaubon. È quello che han fatto tutti, per spiegare i dinosauri e le pesche. (Eco $1988: 678-679$ )

(« Tout au long des flancs du Bricco s’étendent des rangées de vignes. Je les sais, j’en ai vu de semblables de mon temps. Aucune Doctrine des Nombres n'a jamais pu dire si elles lèvent en montée ou en descente. Au milieu des rangées, mais il faut $\mathrm{y}$ marcher pieds nus, le talon un peu calleux, dès l'enfance, il y a des pêchers. Ce sont des pêches jaunes qui ne poussent qu'entre les vignes, elles se fendent sous la pression du pouce, et le noyau en sort presque tout seul, propre comme après un traitement chimique, sauf quelques vermisseaux à la chair grasse et blanche qui y restent attachés par un atome. On peut les manger sans quasiment sentir le velours de la peau, qui vous fait courir des frissons depuis la lange jusqu'à l'aine. Jadis paissaient là les dinosaures. Puis une autre surface a couvert la leur. Et pourtant, comme Belbo au moment où il jouait de la trompette, quand je mordais dans les pêches je comprenais le Royaume et je ne faisais qu'un avec lui. Après, tout n'est qu'un artifice. Invente, invente le Plan, Casaubon. C'est ce qu'ils ont tous fait, pour expliquer les dinosaures et les pêches. ", Eco 1990 : 650)

La «Doctrine des Nombres », une pensée abstraite, n'est pas capable de reconnaître la montée ou la descente ; mais le corps d'un être humain, qui perçoit et joue de la facilité d'une descente ou qui est fatigué et transpire en remontant dans le vignoble, peut immédiatement comprendre ce qui échappe à la Doctrine. Et, dans la suite du texte, on trouve presque tous les sens (sauf l'odorat). La vision, qui saisit les flancs de la colline, la couleur des pêches, le blanc de la chair des vermisseaux (dans l'originel italien le blanc est celui de la pulpe des pêches). Le toucher, atténué dans le talon calleux à force de marcher pieds nus dans les champs, ou stimulé par la pression du doigt sur la pêche et par la surface de velours du fruit. L'ouie, mise en cause du souvenir de la trompette de Belbo. Le goût de la pêche mangée «qui vous fait courir des frissons depuis la langue jusqu'à l'aine ».

32 Casaubon est en train de découvrir, à travers les sens, la vérité qui lui échappait peu avant. Une vérité que la théorie (la Doctrine) ne pourrait lui donner, mais que la narration peut dire au lecteur. Une vérité faite de sensations, de corporéité, d'existence immédiate, hic et nunc. Comme dans le discours gnoséologique, traité par la théorie, était nécessaire un point d'ancrage (le socle dur de l'être), de la même façon le discours existentiel exige son point d'ancrage. Un point d'ancrage matériel, corporel, pour un monde qui, s'il est saisi seulement à travers la connaissance rationnelle, réussit toujours à nous échapper, à se nier, à se retourner contre nous, en se montrant différent de comme nous l'avions pensé. C'est donc une connaissance sensible, esthétique (dans le sens 
originel du terme), celle que Casaubon arrive finalement à retrouver et qui le rend différent de Guillaume, abattu au point de devenir apathique. Guillaume a perdu confiance dans sa sagesse, mais il est encore vif et sera vif pendant beaucoup d'années ; et pourtant, il est désormais abattu. Casaubon est convaincu qu'il mourra bientôt et il perdra tout, mais il a un sursaut final et il retrouve un sens qui le rassérène, une réunion avec «le Royaume ». Et - je répète - c'est un sens matériel, corporel et esthétique. Il a beaucoup été écrit sur la phrase finale du Nom de la rose. Je crois qu'une leçon également importante, et peut-être plus humaine, nous vient des dernières lignes du Pendule de Foucault. «E allora tanto vale star qui, attendere, e guardare la collina È così bella. (Eco $1988: 680$ ) ; (Et alors autant vaut mieux rester ici, attendre, et regarder la colline. Elle est si belle. », ).

\section{BIBLIOGRAPHY}

Beardsworth, S.G. et Auxier, R.E. (2017), éds., The Philosophy of Umberto Eco, The Library of Living Philosophers, vol. 35, Open Court, Chicago.

Bernoussi, M. (2017), Umberto Eco, sémioticien et romancier, Mimésis, Sesto San Giovanni.

Eco, U. (1962), Opera aperta, Bompiani, Milano (trad. fr. L'œuvre ouverte, Points, 2015).

Eco, U. (1975), Trattato di semiotica generale, Bompiani, Milano.

Eco, U. 1980, Il nome della rosa, Bompiani, Milano (trad. fr. Le nom de la rose, Grasset \& Fasquelle, Paris 1982).

Eco, U. 1983, "Postille al "Il nome della rosa" ", in Alfabeta, 49 (trad. fr. Apostille au « Nom de la Rose ", Le livre de poche, Paris 1987).

Eco, U. 1988, Il pendolo di Foucault, Bompiani, Milano (trad. fr. Le pendule de Foucault, Grasset \& Fasquelle, Paris 1990).

Eco, U. 1990, I limiti dell'interpretazione, Bompiani, Milano (trad. fr. Les limites de l'interprétation, Le livre de poche, Paris, 1994).

Eco, U. 1997, Kant e l'ornitorinco, Bompiani, Milano (trad. fr. Kant et l'ornithorynque, Le livre de poche, Paris, 2001).

Eco, U. 2002, Sulla letteratura, Bompiani, Milano (trad. fr. De la literature, Le livre de poche, Paris, 2005).

Eco, U. 2011, Confessions of a Young Novelist, Harvard University Press, Cambridge (trad. fr.

Confessions d'un jeune romancier, Le livre de poche, Paris, 2015).

Eco, U. 2012, « Di un realismo negativo », in De Caro, M. et Ferraris, M., éds., Bentornata realtà, Einaudi, Torino, p. 91-112.

Eco, U. 2017, « Intellectual autobiography », in Beardsworth, S.G. et Auxier, R.E. 2017, p. 3-65.

Giovannoli, R. (1985), éd., Saggi su Il nome della rosa, Bompiani, Milano. 
Hatem, H. 1982, « Le nom de la rose », in Esprit, 69, p. 201-203 (trad. it. «L'eresia nominalista », in Giovannoli 1985, p. 84-87).

Horia, V. 1983, « El nombre de la rosa es politeísmo », in El Alcazar, 9 marzo 1983, p. 30 (trad. it. «Politeismo del Nome della rosa », in Giovannoli 1985, p. 118-121).

Paolucci, C. 2017, Umberto Eco. Tra ordine e avventura, Feltrinelli, Milano.

Polidoro, P. 2012, Umberto Eco e il dibattito sull'iconismo, Aracne, Roma.

Polidoro, P. 2015, « Umberto Eco and the problem of iconism », in Semiotica, 206, p. 129-160.

Polidoro, P. 2017, « The Reasonable's the Limit », in Beardsworth, S.G. et Auxier, R.E. 2017, éds., p. 181-199.

Sommavilla, G. 1981, «L'allegro nominalismo nichilista di Umberto Eco », in La civiltà cattolica, 3150, p. 502-506.

\section{ABSTRACTS}

This article proposes a comparison between the final pages of Umberto Eco's first two novels : The Name of the Rose and The Foucault's Pendulum. In Eco's work, the theoretical activity in semiotics and the writing of novels were narrowly intertwined and narration had a very special role because, as Eco himself used to say, "what you cannot theorize about, you must narrate ». Hence, in order to better understand this comparison, we need to analyze some aspects of Eco's thought. The first aspect is his position about realism in philosophical debate; he supported a specific form of realism, not naïve, which he called "negative». The second aspect is his " reasonable " approach to theoretical discussion. This reasonableness is always linked to the research of an anchor point, a handhold, a clasp, on which to build the rest of the system. It is exactly the presence of this kind of clasp the difference between the finals of the two novels. In The Name of the Rose the lack of an order brings William to a complete defeat, which leaves him in a state of apathy. On the contrary, in The Foucault's Pendulum, in a situation even more dramatic than William's one, Casaubon finally find his serenity, his meaning, in an aesthetic dimension.

L'article propose une comparaison entre les clausules des deux premiers romans d'Umberto Eco : Le Nom de la rose et Le Pendule de Foucault. Dans l'œuvre d'Eco, les activités du théoricien de la sémiotique et du romancier sont étroitement liées car la narration y joue un rôle de premier plan.

Pour mieux comprendre la comparaison entre les deux clausules, donc, il faut analyser certains aspects de la pensée d'Eco. Le premier est la position réaliste qu'il avait dans le débat philosophique ; réalisme particulier, non ingénu, mais défini par lui-même comme "négatif ». Le deuxième aspect est l'approche raisonnable toujours montrée par Eco dans les discussions théoriques. Cette raison est souvent liée à la recherche d'un point d'ancrage, d'un point d'appui sur lequel fonder le reste de son système.

C'est exactement la présence d'un tel point d'ancrage qui différencie les deux clausules. Dans Le Nom de la rose, en effet, l'absence d'un ordre conduit Guillaume à une défaite totale, qui le laisse dans un état d'apathie; par contre, dans Le Pendule de Foucault, dans une situation bien plus dramatique que celle de Guillaume, Casaubon finalement trouve son point de sérénité, son sens dans la dimension esthétique. 
INDEX

Keywords: Umberto Eco, The Name of the Rose, Foucault's Pendulum, realism, semiotics, narration

Mots-clés: Umberto Eco, Le Nom de la rose, Le Pendule de Foucault, réalisme, sémiotique, narration

\section{AUTHOR}

PIERO POLIDORO

Université LUMSA (Rome) 